\title{
Damage and Failure Process of Concrete Structure under Uniaxial Compression Based on Peridynamics Modeling
}

\author{
Feng Shen, Qing Zhang, and Dan Huang \\ Department of Engineering Mechanics, Hohai University, Nanjing 210098, China \\ Correspondence should be addressed to Qing Zhang; lxzhangqing@hhu.edu.cn
}

Received 19 July 2013; Accepted 18 September 2013

Academic Editor: Zhiqiang $\mathrm{Hu}$

Copyright (C) 2013 Feng Shen et al. This is an open access article distributed under the Creative Commons Attribution License, which permits unrestricted use, distribution, and reproduction in any medium, provided the original work is properly cited.

\begin{abstract}
Peridynamics is a nonlocal formulation of continuum mechanics, which uses integral formulation rather than the spatial partial differential equations. The peridynamic approach avoids using any spatial derivatives, which arise naturally in the classical local theory. It has shown effectiveness and advantage in solving discontinuous problems at both macro- and microscales. In this paper, the peridynamic theory is used to analyze damage and progressive failure of concrete structures. A nonlocal peridynamic model for concrete columns under uniaxial compression is developed. Numerical example illustrates that cracks in a peridynamic body of concrete form spontaneously. The result of the example clarifies the unique advantage of modeling damage accumulation and progressive failure of concrete based on peridynamic theory. This study provides a new promising alternative for analyzing complicated discontinuity problems. Finally, some open problems and future research trends in peridynamics are discussed.
\end{abstract}

\section{Introduction}

Concrete is a complex material which is used widely in civil engineering structures such as dams, buildings, and bridges. Concrete is difficult to model because it is a quasibrittle material, and precise analysis of concrete structures is elusive. In fact, concrete exhibits many discontinuities even before the application of load. Expansion, shrinkage, and temperature changes often cause cracking. The formation of cracks significantly affects the stress and displacement fields. Even though concrete has been studied for many years, its progressive failure process, especially the mechanism of spontaneous formation of a crack, and its subsequent growth have not been fully understood yet. The damage of concrete is the process of crack initiation, propagation, and coalescence, which may cause a sudden collapse suddenly. Over the past several decades, continuum mechanics and finite element method (FEM) solved many problems in solid mechanics. The finite element method has become the most commonly accepted technique for the numerical solution of structural problems. Continuum mechanics and finite element method start with an assumption of a spatially continuous and differentiable displacement field. Strains are obtained from spatial derivatives of displacement field. When applied to concrete, they have limited application to model damage. As mentioned earlier, problems appear when damage is involved. The basic assumption of displacement continuity is not valid. Nearly all traditional methods based on the classical theory of continuum mechanics attempt to solve the partial differential equations that include derivatives of the displacement components, but these derivatives are undefined when the displacements are discontinuous, such as across cracks or interfaces.

To overcome the shortcoming of the classical theory of continuum mechanics, Silling, at Sandia Nation Laboratories, introduces the peridynamic theory [1], which does not assume spatial differentiability of displacement field and permits discontinuities to arise as part of solution. The term "peridynamic" comes from the Greek words "peri" and "dynamic," which mean "near" and "force," respectively. This theory is nonlocal, which can be thought of as a generalization of classical theory of elasticity.

After mathematical demonstration of the theory, peridynamics has been applied to $1 \mathrm{D}$ bar [2], membranes, fibers [3], and so on. However, peridynamic theory has not been applied to model concrete very often. Gerstle et al. $[4,5]$ 


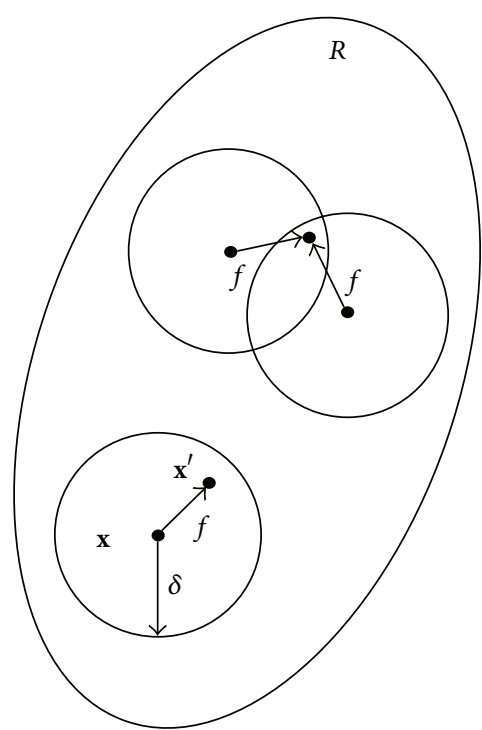

FIGURE 1: Pairwise interaction of a material point with its neighboring points.

model the damage of concrete structures using a simple conceptual peridynamic model, and Kilic and Madenci [6] have done some research on the structural stability of a concrete column. Shen et al. [7, 8] and Huang et al. [9, 10] employed peridynamic models to simulate the damage and progressive failure of the concrete and clarified the unique advantage of peridynamics.

This paper aims to investigate the capability of peridynamic to model concrete structure under uniaxial compression. The remaining of the paper is organized as follows. In Section 2, a brief introduction to peridynamic theory and the solution method used in the present work is given. A description of the peridynamic model for uniaxial compression of concrete and the simulation results are presented in Section 3. Then some concluding remarks and the shortcomings of peridynamic theory are finally provided at the end of this paper.

\section{Peridynamic Theory and Solution Method}

The peridynamic theory was first introduced by Silling [1] in 2000, which makes no assumption of continuity of displacements. In peridynamics, the material domain is discretized into an infinite number of infinitesimal interacting particles. Particles separated by a finite distance can interact with each other. The maximum interaction distance between particles in peridynamics is called the "material horizon." When the distance between two particles is less than the material horizon, they interact with each other; otherwise they do not interact. The interacting forces between particles are calculated through empirical particle interaction relations. Particles move in accordance with Newton's second law.
Referring to Figure 1, the peridynamic formulation proposed by Silling [1] is defined by the following equation:

$$
\begin{array}{r}
L_{\mathbf{u}}(\mathbf{x}, t)=\int_{R} f\left(\mathbf{u}\left(\mathbf{x}^{\prime}, t\right)-\mathbf{u}(\mathbf{x}, t), \mathbf{x}^{\prime}-\mathbf{x}\right) d V_{\mathbf{x}^{\prime}}, \\
\forall \mathbf{x} \in R, t \geq 0 .
\end{array}
$$

In this formulation, at any point $x$ in the reference configuration, $R$, at any time $t$, each pair of particles interacts through a force function $f$. $L$ is the pair wise force acting upon $\mathbf{x}$ due to all particles $\mathbf{x}^{\prime}$ within its horizon, $u$ is the displacement field, and $d V_{\mathbf{x}^{\prime}}$ is the integration variable that indicates infinitesimal domain located at point $\mathbf{x}^{\prime}$. According to Newton's second law, the peridynamic equation of motion is given by

$$
\rho \frac{\partial^{2} \mathbf{u}}{\partial t^{2}}=\int_{H} f\left(\mathbf{u}\left(\mathbf{x}^{\prime}, t\right)-\mathbf{u}(\mathbf{x}, t), \mathbf{x}^{\prime}-\mathbf{x}\right) d V_{\mathbf{x}^{\prime}}+\mathbf{b}(\mathbf{x}, t),
$$

where $\mathbf{b}$ is a prescribed body-force density field which represents the external force per unit reference volume, $\rho$ is mass density in the reference configuration, and $H$ is the neighborhood of material points $\mathbf{x}$ within the horizon size of $\delta$ as follows:

$$
H=H(\mathbf{x}, \delta):=\left\{\mathbf{x}^{\prime} \in R:\left\|\mathbf{x}^{\prime}-\mathbf{x}\right\| \leq \delta\right\} .
$$

$\delta$ is a positive value, called the material horizon. When the distance between two particles is less than or equal to $\delta$, they will interact with each other through the force function $f$; otherwise, if the distance exceeds horizon, the interaction may vanish. In calculation, the value of horizon size $\delta$ can be confirmed according to the requirement of simulation efficiency and precision. It can be found that a much larger horizon size will lead to a higher accuracy but lower efficiency. Referring to the literatures [11, 12] and practices for macroscale modeling, we choose the horizon which is three times the lattice constant. And $f$ is the pairwise force between particles $\mathbf{x}$ and $\mathbf{x}^{\prime}$, which depends on the relative position and the relative displacement between the two particles. Silling uses $\boldsymbol{\xi}, \boldsymbol{\eta}$ for the relative position and the relative displacement vectors, respectively as follows:

$$
\xi=\mathbf{x}^{\prime}-\mathbf{x}, \quad \boldsymbol{\eta}=\mathbf{u}^{\prime}-\mathbf{u} .
$$

Therefore, the vector $\boldsymbol{\eta}+\boldsymbol{\xi}=\left(\mathbf{x}^{\prime}+\mathbf{u}^{\prime}\right)-(\mathbf{x}+\mathbf{u})$ denotes the current relative position between points $\mathbf{x}^{\prime}$ and $\mathbf{x}$ in the deformed configuration. This means that the force vector between two particles is parallel to their relative current position vector. The pairwise function $f$ contains all of the constitutive information of the material. Because the interaction of particles is at a finite distance, peridynamic is in the category of nonlocal models. Peridynamics is not defined in the form of stress and strain, and it eliminates the assumption on the continuity or differentiability of the displacement field or small-deformation behavior thoroughly. Many studies [13-15] proposed various peridynamic constitutive models combined with the constitutive information of the material in the force function $f$. Any function satisfying the linear and 
the angular admissibility condition is a valid force function. For a bond-based peridynamic linear elastic material, the pairwise force function $f$, is given by [11]

$$
f(\boldsymbol{\eta}, \boldsymbol{\xi})=c \mathbf{s}
$$

where $f$ has units of force per unit volume squared, $c$ is called the peridynamic microelastic constant, and $\mathbf{s}$ is the bond stretch as follows:

$$
\mathbf{s}=\frac{|\xi+\eta|-|\xi|}{|\xi|}
$$

Regardless of the distance among interacting points, the force function $f$ assumes that the interaction force remains the same for a constant stretch. Considering the effect of thermal loading, (5) can be modified as

$$
f(\boldsymbol{\eta}, \boldsymbol{\xi})=c(\mathbf{s}-\alpha \theta) ;
$$

$\alpha$ and $\theta$ stand for the coefficient of thermal expansion and the temperature difference between current and reference temperatures between material points $\mathbf{x}^{\prime}$ and $\mathbf{x}$. According to the prototype microelastic brittle (PMB) model introduced by Silling and Askari [12], we have

$$
c=\frac{18 K}{\pi \delta^{4}}
$$

where $K$ is the bulk modulus of the material expressed in terms of elastic modulus, $E$ and Poisson's ratio, $v$ as

$$
k=\frac{E}{3(1-2 \nu)} .
$$

So the peridynamic equation of motion (2) can be rewritten as

$$
\rho \frac{\partial^{2} \mathbf{u}}{\partial t^{2}}=\int_{H_{\mathbf{x}}} \mathbf{f}(\boldsymbol{\eta}, \boldsymbol{\xi}) d V_{\boldsymbol{\xi}}+\mathbf{b}(\mathbf{x}, t)
$$

with

$$
\mathbf{f}(\boldsymbol{\eta}, \xi)=f(\boldsymbol{\eta}, \xi) \frac{\boldsymbol{\xi}+\boldsymbol{\eta}}{|\boldsymbol{\xi}+\boldsymbol{\eta}|},
$$

where $H_{\mathrm{x}}$ is the neighborhood of the particle $x$; it is assumed to be a spherical region centered at $\mathbf{x}$ with the radius of the material horizon, and the horizon is analogous to the cutoff radius used in molecular dynamics. follows:

The break of the bond is described by the factor $\mu$ as

$$
\mu(t, \xi)= \begin{cases}1, & s\left(t^{\prime}, \xi\right)-\alpha \theta<s_{0}, 0 \leq t^{\prime} \leq t \\ 0, & \text { otherwise }\end{cases}
$$

where $s_{0}$ is a critical stretch of the material for failure. Although $s_{0}$ is expressed as a property of a particle, bond breaking must be a symmetric operation for all particle pairs sharing a bond [12]. Therefore, we can define the damage at a point $\mathbf{x}$ as

$$
\phi(\mathbf{x}, t)=1-\frac{\int_{H_{\mathbf{x}}} \mu(\boldsymbol{\eta}, \boldsymbol{\xi}) d V_{\mathbf{x}^{\prime}}}{\int_{H_{\mathbf{x}}} d V_{\mathbf{x}^{\prime}}} .
$$

To solve the peridynamic equation of motion, the region defining a peridynamic material is discretized into particles (material points), forming a simple cubic lattice with the lattice constant $\Delta \mathbf{x}$, and a numerical approximation is usually implemented by reducing the spatial integration into finite sum. So the integral in (2) is replaced by

$$
\rho \ddot{\mathbf{u}}_{\mathbf{i}}^{n}=\sum_{p \in \mathrm{Q}} \mathbf{f}\left(\mathbf{u}_{p}^{n}-\mathbf{u}_{i}^{n}, \mathbf{x}_{p}-\mathbf{x}_{i}\right) V_{p}+\mathbf{b}_{i}^{n},
$$

where $n$ is the number of time steps, subscripts denote the particle number, and $Q$ represents the family of particles for which particle $\mathbf{x}$ shares a bond in the reference configuration within the horizon. That is,

$$
Q=\left\{p \mid\left\|\mathbf{x}^{\prime}-\mathbf{x}\right\| \leq \delta\right\} .
$$

Through calculation test considering both efficiency and stability, the appropriate time step size for different simulation task can be confirmed [12].

\section{Numerical Implementation and Results}

In the following, a concrete column with the diameter of $0.15 \mathrm{~m}$ is presented. The height of the column is $0.3 \mathrm{~m}$. The concrete material considered is assumed to be homogeneous, isotropic, and brittle elastic, and the elastic constants are identical to those in classical continuum model. The concrete has density $\rho=2200 \mathrm{~kg} / \mathrm{m}^{3}$. The bulk modulus $K=20 \mathrm{GPa}$, and a critical bond stretch for failure of a bond is set as $\mathbf{s}_{0}=0.005 \mathrm{~m}$. In the peridynamic simulation, the column is discretized into particles forming a simple cubic lattice with lattice constant $\Delta \mathbf{x}=0.005 \mathrm{~m}$ and horizon $0.015 \mathrm{~m}$. (The horizon is three times the lattice constant.) The temperature of the whole system is kept at $300 \mathrm{~K}$.

The position and velocity of each particle are calculated as a solution to the equation of motion using the velocityvelert time integration algorithm, and the time step is set to $1 \times 10^{-7} \mathrm{~s}$, based on the combined optimization test of both integration stability and computation efficiency [12].

The numerical experiment was performed to simulate the damage and progressive failure of the concrete column under uniaxial compression. In the simulation, the velocity of all particles was initially set to zero along the three orthogonal directions in Cartesian coordinates. When the uniaxial deformation simulation is implemented, the ten layers of particles at the bottom of column are constrained to be fixed with a zero velocity, as the top ten layers of particles were set to have a downward velocity of $20 \mathrm{~m} / \mathrm{s}$. Figure 2 shows the damage and failure process of the concrete column under uniaxial compression. It can be found that the break of bonds appears symmetrically. The top ten layers of particles have moved down during the simulation, and the specimen has formed the diagonal shear bands, as well as the dynamic ejection of crushed material from the middle of the column. Even more the fracture tracks along the direction of $45^{\circ}$ above horizon which is consistent with the experimental results of concrete structures under uniaxial compression. Figure 3 displays the failure mode of concrete column section under uniaxial compression using peridynamic model. 


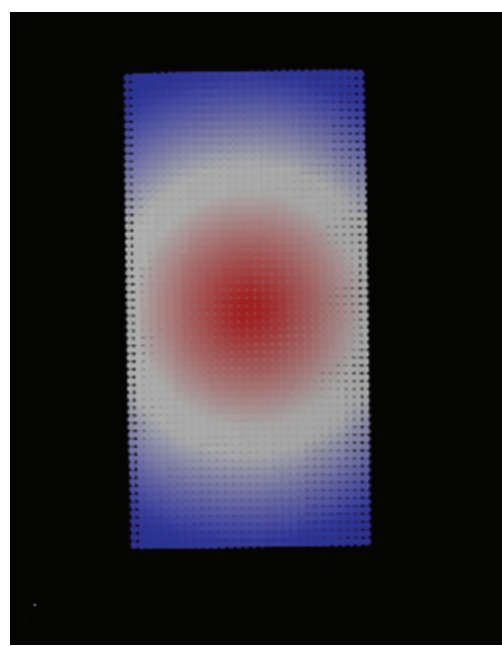

(a)

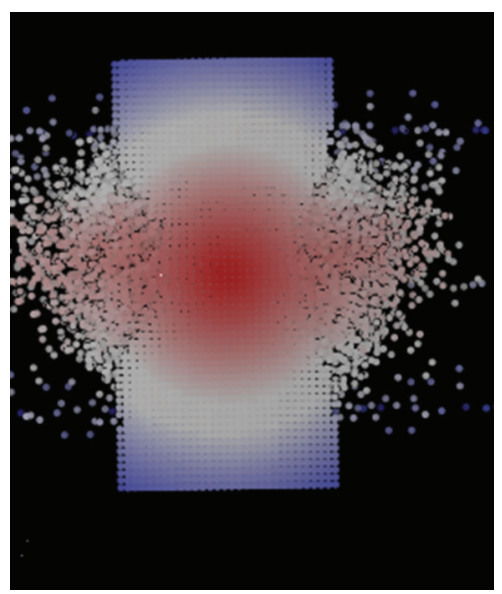

(d)

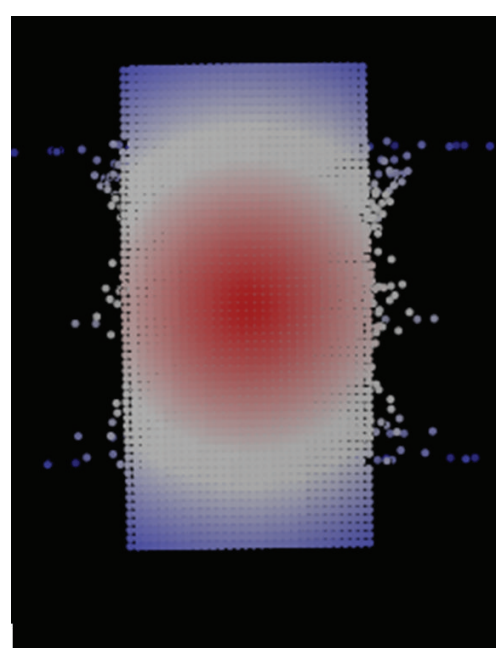

(b)

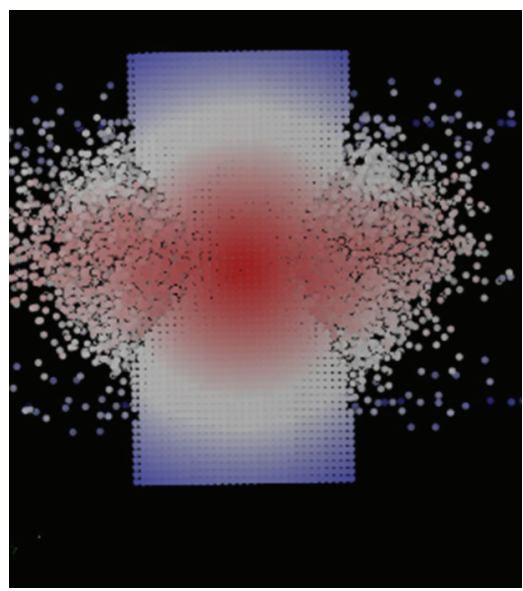

(e)

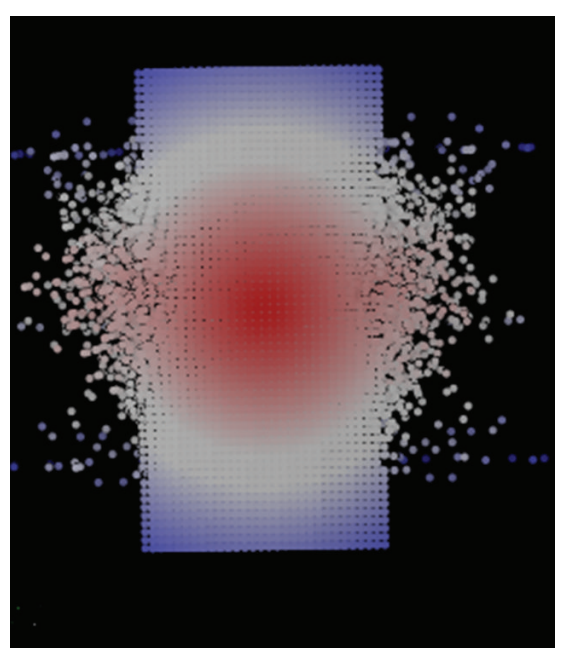

(c)

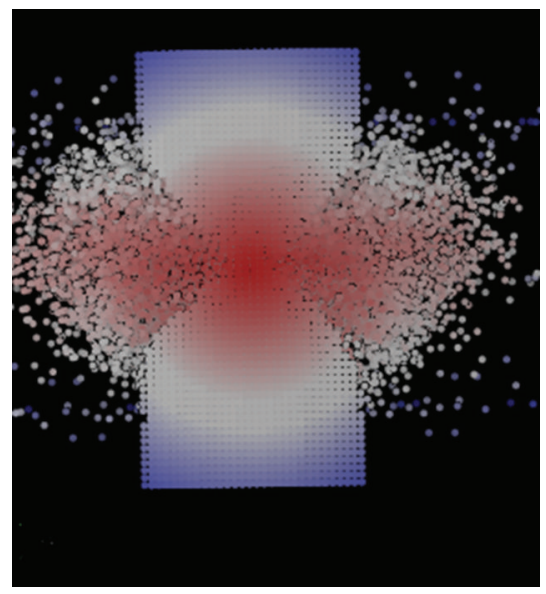

(f)

FIGURE 2: Peridynamic simulation of concrete column subjected by uniaxial compression, where $t=1000,2000,3000,4000,5000,6000$ time steps for (a) to (f), respectively.

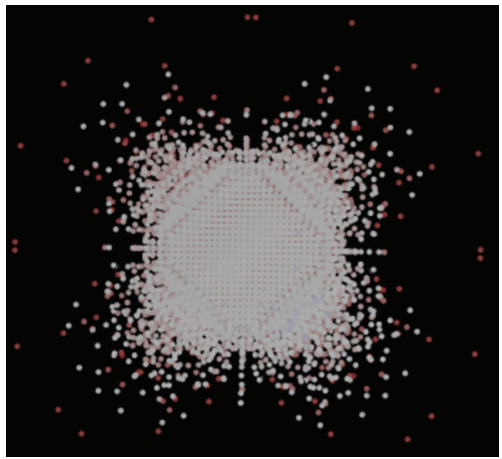

FIGURE 3: Peridynamic simulation of the failure mode of concrete column under uniaxial compression.

From the simulation results, it is observed that the damage and failure of the column is just spontaneous, and no special failure criteria are required in order to predict the path along which the crack develops.

\section{Concluding Remarks}

This paper takes initial attempt to solve the concrete column under uniaxial compression based on the peridynamic theory. The result of the study clarifies the unique advantage of peridynamics in modeling concrete structures. As a reformation of the classical continuum mechanics theories, peridynamics is a powerful tool to solve discontinuities problems. The peridynamic theory can avoid singularity in solving discontinuity problems by integral equations rather than differential equations. The basic equations can be applied anywhere in the model including at the cracks, so no additional technique is necessary for the study of damage and cracking. The problem of concrete column under uniaxial compression shows that a relatively simple peridynamic model can simulate the entire damage evolution and fracture process in concrete structures effectively with great potential over both mesh free method and molecular dynamics. In other words, the peridynamic model can be regarded as a coarse-grain molecular dynamics model. The 
pairwise function contains all of the constitutive information of the material, and the limitations such as remeshing and mapping can be removed thoroughly.

As a new powerful tool, the peridynamic theory has some shortcomings itself. Dealing with an infinite number of material particles, a much greater calculation time is required. As each particle will interact with its neighbors within the horizon besides the nearest ones, the peridynamic simulation at a nondamaged region is computationally expensive compared with Finite Element Method. In addition to this, the original peridynamic constitutive models oversimplify the interaction between the particles in the sense that the mutual force between two particles is assumed to be independent of the positions of other particles within the horizon. The rotation of the particles is not considered, which influence the exactitude of the constitutive relations of the material. The plasticity and viscosity in the classical theory of continuum mechanics based on peridynamics are still under research, which limits the application. And more efficient solution algorithms and appropriate peridynamic constitutive models need to be developed. Furthermore, a multiscale research approach combining peridynamics with molecular dynamics and finite element method will be a promising way to solve discontinuous problems more accurately and effectively. And the boundary and the size effects arising in the peridynamic model need to be investigated in the future.

\section{Conflict of Interests}

The authors of the paper declare that there is no conflict of interests regarding the publication of this paper. The authors do not have a direct financial relation with the commercial identity that might lead to a conflict of interests for any of the authors.

\section{Acknowledgment}

This work was supported by the National Nature Science Foundation of China (Grant nos. 11372099, 51179064, 11132003, and 51209079) and the Graduate Students Research and Innovation Plan of Jiangsu Province (no. CXZZ11_0425).

\section{References}

[1] S. A. Silling, "Reformulation of elasticity theory for discontinuities and long-range forces," Journal of the Mechanics and Physics of Solids, vol. 48, no. 1, pp. 175-209, 2000.

[2] S. A. Silling, M. Zimmermann, and R. Abeyaratne, "Deformation of a peridynamic bar," Journal of Elasticity, vol. 73, no. 1-3, pp. 173-190, 2003.

[3] S. A. Silling and F. Bobaru, "Peridynamic modeling of membranes and fibers," International Journal of Non-Linear Mechanics, vol. 40, no. 2-3, pp. 395-409, 2005.

[4] W. Gerstle, N. Sau, and E. Aguilera, "Peridynamic modeling of plain and reinforced concrete structures," in Proceedings of the 18th International Conference on Structural Mechanics in Reactor Technology (SMiRT '05), Beijing, China, 2005.

[5] W. Gerstle, N. Sau, and S. Silling, "Peridynamic modeling of concrete structures," Nuclear Engineering and Design, vol. 237, no. 12-13, pp. 1250-1258, 2007.
[6] B. Kilic and E. Madenci, "Structural stability and failure analysis using peridynamic theory," International Journal of Non-Linear Mechanics, vol. 44, no. 8, pp. 845-854, 2009.

[7] F. Shen, Q. Zhang, D. Huang, and J. Zhao, "Peridynamics modeling of failure process of concrete structure subjected to impact loading," Engineering Mechanics, vol. 29, no. s1, pp. 12$15,2012$.

[8] F. Shen, Q. Zhang, D. Huang, and J. Zhao, "Damage and failure process of concrete structure under uni-axial tension based on peridynamics modeling," Chinese Journal of Computational Mechanics, vol. 30, pp. 79-83, 2013.

[9] D. Huang, Q. Zhang, and P. Z. Qiao, "Damage and progressive failure of concrete structures using non-local peridynamic modeling," Science China Technological Sciences, vol. 54, no. 3, pp. 591-596, 2011.

[10] D. Huang, Q. Zhang, P. Qiao, and F. Shen, "A review on peridynamics method and its applications," Advances in Mechanics, vol. 40, no. 4, pp. 448-459, 2010.

[11] M. L. Parks, R. B. Lehoucq, S. J. Plimpton, and S. A. Silling, "Implementing peridynamics within a molecular dynamics code," Computer Physics Communications, vol. 179, no. 11, pp. 777-783, 2008.

[12] S. A. Silling and E. Askari, "A meshfree method based on the peridynamic model of solid mechanics," Computers and Structures, vol. 83, no. 17-18, pp. 1526-1535, 2005.

[13] Q. Du and K. Zhou, "Mathematical analysis for the peridynamic nonlocal continuum theory," ESAIM: Mathematical Modelling and Numerical Analysis, vol. 45, no. 2, pp. 217-234, 2011.

[14] B. K. Tuniki, Peridynamic constitutive model of concrete [M.S. thesis], University of New Mexico, 2012.

[15] P. Seleson and M. L. Parks, "On the role of the influence function in the peridynamic theory," International Journal for Multiscale Computational Engineering, vol. 9, no. 6, pp. 689-706, 2011. 


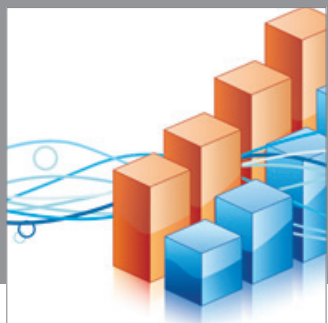

Advances in

Operations Research

mansans

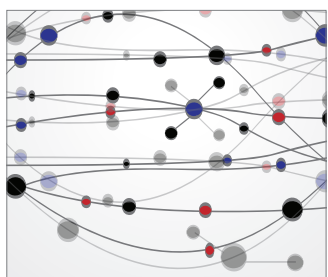

The Scientific World Journal
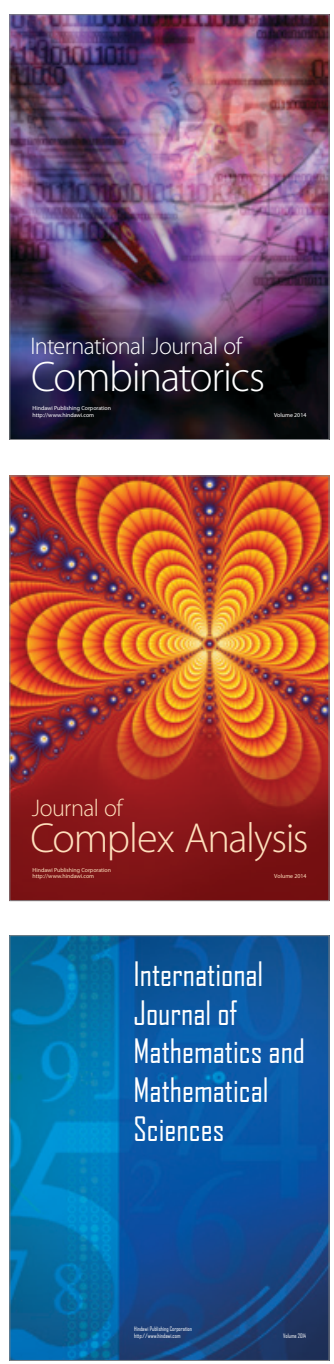
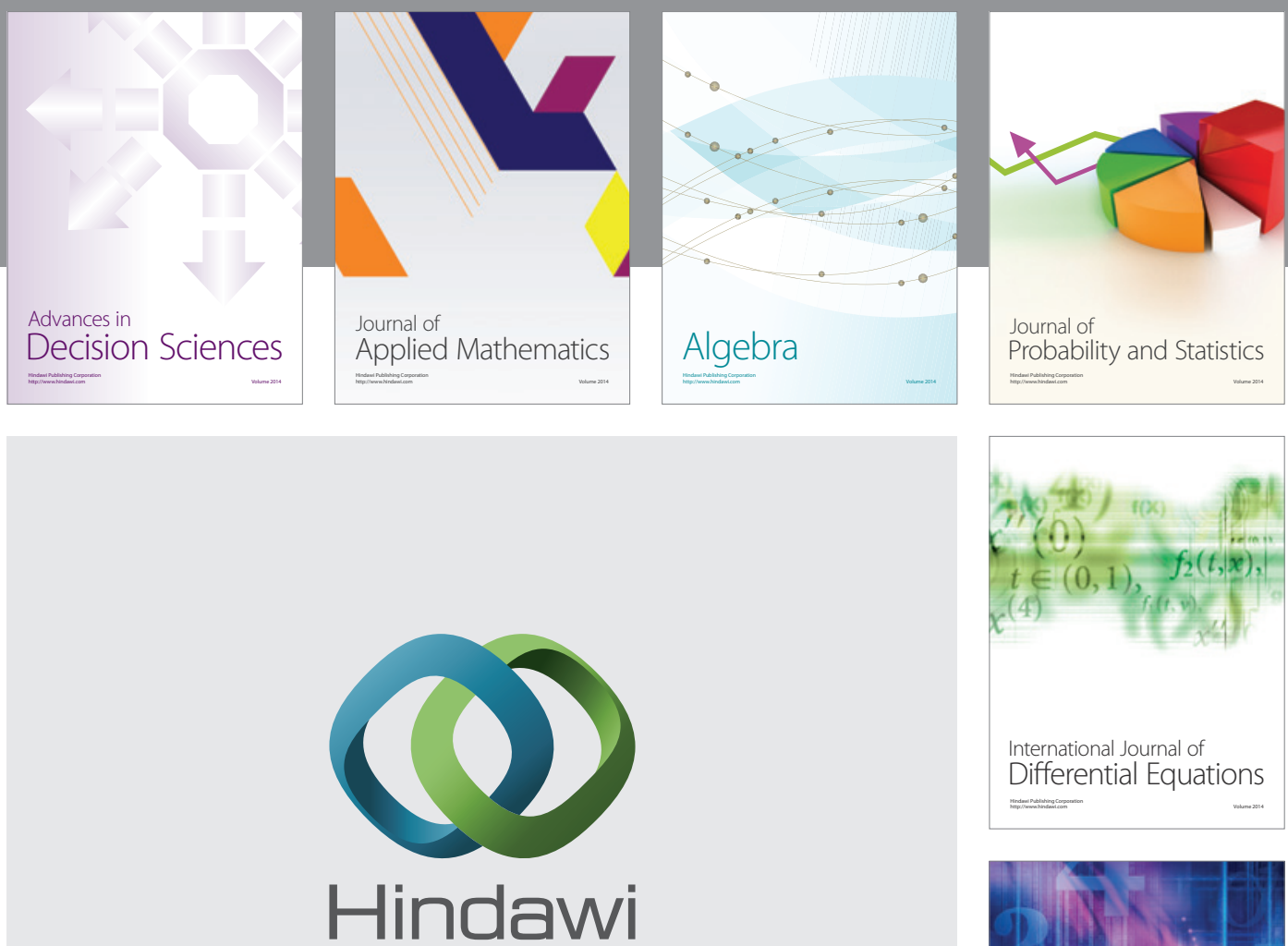

Submit your manuscripts at http://www.hindawi.com
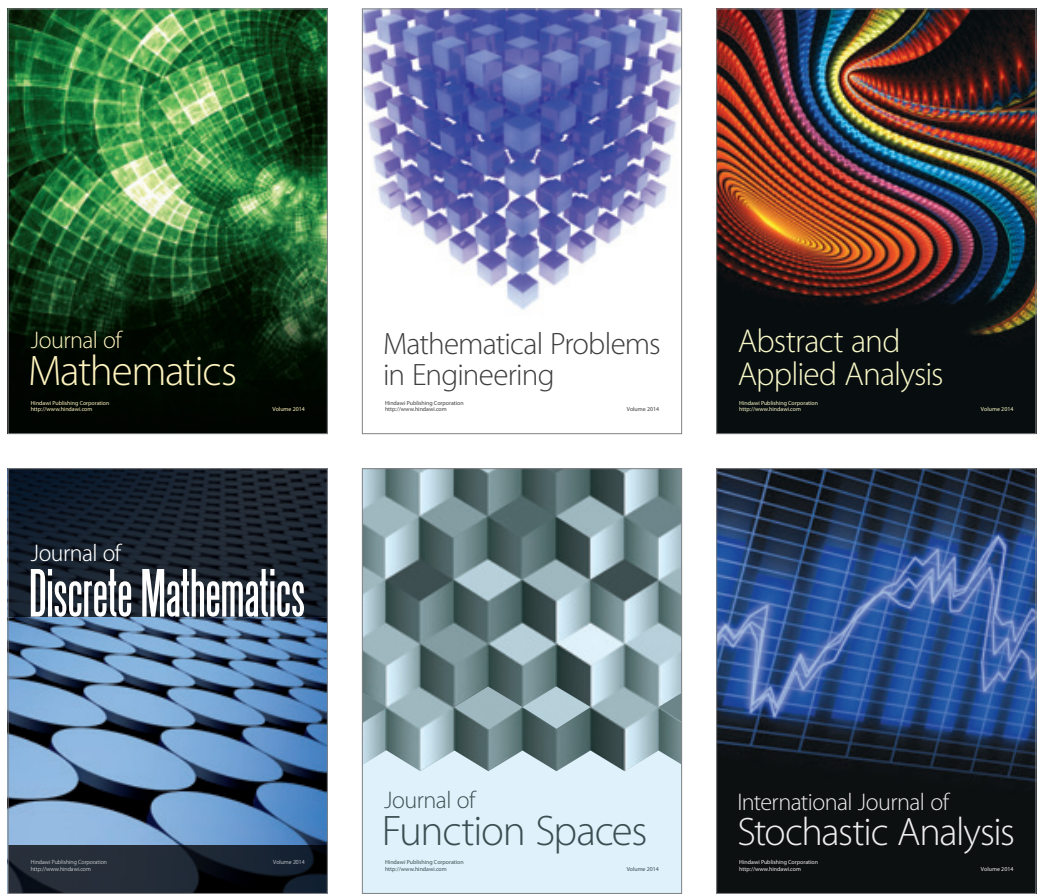

Journal of

Function Spaces

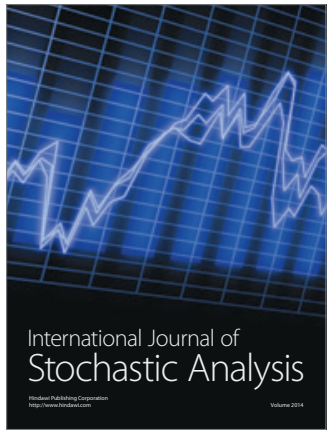

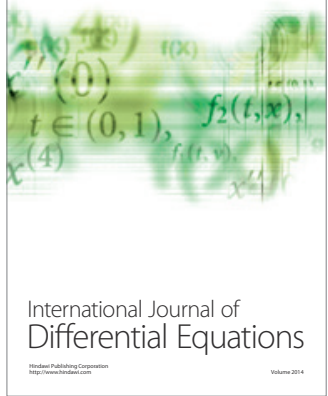
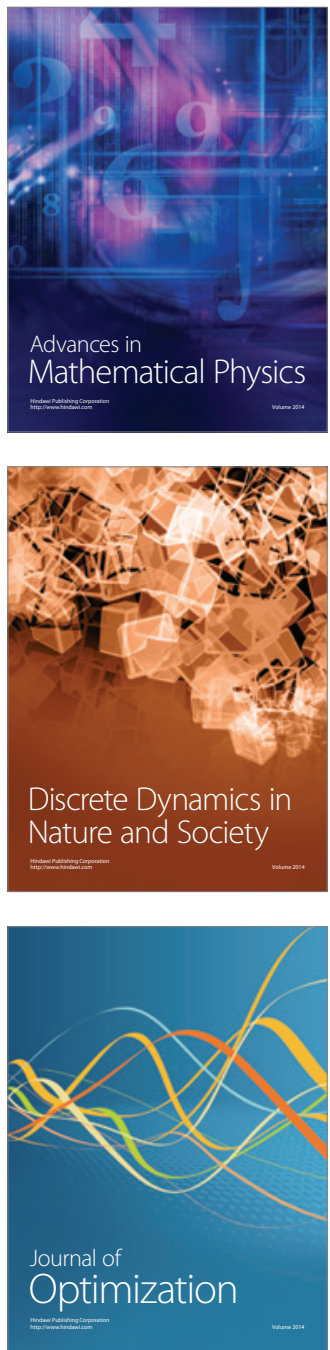